\title{
GENERALIZED FUNCTIONS ASYMPTOTICALLY HOMOGENEOUS WITH RESPECT TO ONE-PARAMETRIC GROUP AT ORIGIN
}

\author{
YU.N. DROZHZHINOV, B.I. ZAVIALOV
}

\begin{abstract}
In the work we obtain a complete description of generalized functions asymptotically homogeneous at origin along a multiplicative one-parametric group of transformations so that the real parts of all the eigenvalues of its infinitesimal matrix are positive including the case of critical orders. The obtained results are applied for constructing asymptotically homogeneous solutions to differential equations whose symbols are quasihomogeneous polynomials along this group in the non-critical case.
\end{abstract}

Keywords: generalized functions, homogeneous functions, quasi-asymptotics, partial differential equations.

\section{INTRODUCTION}

This work is a generalization of our paper [1]. Let $U=\left\{U_{k}, k>0\right\}$ be a multiplicative one-parametric group of linear transformations in $\mathbb{R}^{n}$, so that $U_{k_{1} k_{2}}=U_{k_{1}} U_{k_{2}}$, and we assume that the real parts of the eigenvalues of the group generator are positive. Also let $\mathcal{S}$ be some space of test functions $\left(S\left(R^{n}\right), \mathcal{D}\left(R^{n}\right)\right.$, etc.) invariant w.r.t. $U_{k}, \varrho(k)$ be a positive continuous function on $k>0$, and $f \in \mathcal{S}^{\prime}$ (as usually, by prime we denote the space of the associated generalized functions).

Definition 1. We say that $f$ has a quasiasymptotics at zero (at infinity) w.r.t. $\rho(k)$ along the group $U_{k}$, if for each $\psi(t) \in \mathcal{S}$ and some $g \in \mathcal{S}^{\prime}$

$$
\frac{1}{\varrho(k)}\left(f\left(U_{\frac{1}{k}} t\right), \psi(t)\right) \underset{k \rightarrow \infty}{\longrightarrow}(g(t), \psi(t))
$$

$$
\left(\frac{1}{\rho(k)}\left(f\left(U_{k} t\right), \psi(t)\right) \underset{k \rightarrow \infty}{\longrightarrow}(g(t), \psi(t))\right) .
$$

In this case one also says that $f$ is asymptotically homogeneous on $\mathcal{S}$ along the group $U=$ $\left\{U_{k}, k>0\right\}$ at zero (at infinity) and writes $f \in A O_{\varrho}^{-U}(\mathcal{S})\left(f \in A O_{\varrho}^{U}(\mathcal{S})\right.$, respectively). In the one-dimensional case when $U_{k}$ is the multiplication by $k$ we shall write $f \in A O_{\varrho}^{-1}(\mathcal{F})$ and $f \in A O_{\varrho}^{1}(\mathcal{S})$, respectively.

If $g \equiv 0$, we say that $f(t)$ possesses trivial quasiasymptotics in group $U$. If $f \in \mathcal{S}^{\prime}$ satisfies relation 1.1 and $g \not \equiv 0$, the function $\varrho(k)$ is necessary self-similar (regularly varying) function.

Yu. N. Drozhzhinov, B.I. Zavialov, Generalized functions asymptotically homogeneous along one-parametric group at origin.

(c) Drozhzhinov Yu.N., Zavialov B.I. 2013.

The work is financially supported by RFBR, grant no. 10-01-00178, and the grant of President of Russia for support of Leading Scientific Schools NSh-2928.2012.1.

Submitted April 25, 2012. 
We remind that a positive continuous function $\varrho(k), k>0$, is called self-similar, if for each $a>0$ and some $\alpha \in \mathbb{R}$

$$
\frac{\varrho(a k)}{\varrho(k)} \underset{k \rightarrow \infty}{\longrightarrow} a^{\alpha}
$$

uniformly on compacts in $a$, cf. [6]. The number $\alpha$ is called an order of self-similarity $\varrho$. The order $\alpha$ of the self-similar function $\varrho(k)$ appearing in $(1.1)$ is called an order of asymptotically homogeneous generalized function. We note that each self-similar function $\varrho(k)$ of order $\alpha$ can be represented as

$$
\varrho(k)=k^{\alpha} L(k), \quad k>0,
$$

where $L(k)$ is a self-similar function of zero order (slowly varying function). We admit a complex order of self-similarity (and thus complex self-similar functions) bearing in mind that a complex self-similar function has representation $(1.2)$ with $\alpha \in \mathbb{C}$.

We observe that if $\varrho(k)$ in relation $(1.1)$ is of order $\alpha$, then $g$ is a homogeneous generalized function of power $\alpha \in \mathbb{C}$ in corresponding group of argument transformations

$$
g\left(U_{k} t\right)=k^{\alpha} g(t), \quad k>0 .
$$

Sometimes such functions are called "quasihomogeneous" of order $\alpha$ w.r.t. group $U$, cf. [8].

Asymptotically homogeneous functions are well-studied in the space $S_{+}^{\prime}$ of generalized functions from Schwarz space $S^{\prime}$ with supports on positive semiaxis. A function $f(r) \in S_{+}^{\prime}$ is asymptotically homogeneous at zero w.r.t. self-similir function $\rho(k)$ of order $\alpha$, if

$$
\frac{1}{\rho(k)} f\left(\frac{r}{k}\right) \underset{k \rightarrow \infty}{\longrightarrow} C f_{-\alpha+1}(r) \quad \text { in } S_{+}^{\prime},
$$

where $f_{N}(r)$ is the kernel of fractional Liouville (differentiation) integration. We recall that $f(r)$ is asymptotically homogeneous at zero w.r.t. self-similar function $\rho(k)$ of order $\alpha$ if and only if there exists a number $N>-\alpha+1$, such that its $N$ th primitive is continuous and has usual asymptotics w.r.t. $r^{N} \rho\left(\frac{1}{r}\right)$.

We note that $U_{k}$ can be represented as $U_{k}=e^{\ln k E}$, where $E$ is some linear transformation $\mathbb{R}^{n}$. In works [3], 4] there was provided the description of asymptotically homogeneous at infinity (at zero in [1]) generalized function in the case when $E$ is strictly diagonal, its eigenvalues are real and of the same sign. In a particular case, when the eigenvalues of the matrix $E$ are also same (the corresponding transformation group is the group of dilations of $\mathbb{R}^{n}$ ), the complete description of homogeneous generalized functions along such group is provided in [2].

The main aim of the present work is to obtain the complete description of generalized functions asymptotically homogeneous at zero in a group of multiplicative one-parametric transformations for which real parts of all the eigenvalues of infinitesimal matrix of group $U$ are positive. At that, together with a normal component, in the matrix $E$ there can be also a nilpotent part. As the main tool of such description serves so-called generalized spherical representation of generalized functions [5] described in the second section. This representation reduces the study of asymptotic properties of generalized function at zero w.r.t. the group $\left\{U_{k}, r>0\right\}$ to studying radial asymptotic properties of generalized functions defined on special spaces of test functions.

Asymptotically homogeneous generalized functions on these special spaces are studied in Section 3. There we also give a description of generalized functions in $S^{\prime}\left(\mathbb{R}^{n}\right)$ asymptotically homogeneous along the trajectories determined by the multiplicative one-parametric group. We note that in Section 3 we give some statements without proofs since their main ideas they are close to the proofs of similar statements in [1], [5] and can be easily reproduced in the new situation.

Finally, in the last Section we prove the theorem on division of a generalized function by a polynomial homogeneous w.r.t. group $U_{k}$ and the obtained results are applied for constructing 
asymptotically homogeneous solutions to differential equations, whose symbols are homogeneous polynomials, in noncritical case.

\section{Generalized SPHERICAl REPRESEntation of GENERALIZED FUnCtions}

Generalized spherical representation in the most appropriate for us form was introduced in [5]. For the reader's convenience we reproduce the main milestones of its construction.

Suppose in $\mathbb{R}^{n}$ (and hence in $\mathbb{C}^{n}$ ) there acts a real continuous multiplicative group of linear transformations $U=\left\{U_{k}=e^{\ln k E}, k>0\right\}$. The operator $E$ being the generator of this group is represented as

$$
E=H+N ; \quad H=M+i L,
$$

where $H$ is normal and $N$ is nilpotent component of this operator. The operator $H$ reads as $\sum_{j} \kappa_{j} \mathcal{P}_{j}$, where $\kappa_{j}$ are its eigenvalues and $\mathcal{P}_{j}$ are the projections on the associated eigenspaces. At that, $M=\sum_{j} \operatorname{Re} \kappa_{j} \mathcal{P}_{j}$, and $L=\sum_{j} \operatorname{Im} \kappa_{j} \mathcal{P}_{j}$. We observe that all these operators commute. The one-parametric groups associated with these operators are denoted as

$$
\mathfrak{H}_{k}=e^{\ln k H} ; \quad \mathfrak{M}_{k}=e^{\ln k M} ; \quad \mathfrak{L}_{k}=e^{i \ln k L} ; \quad \mathfrak{N}_{k}=e^{\ln k N},
$$

so that

$$
U_{k}=\mathfrak{H}_{k} \cdot \mathfrak{N}_{k}=\mathfrak{M}_{k} \cdot \mathfrak{L}_{k} \cdot \mathfrak{N}_{k}
$$

Let

$$
\sigma=\left(\sigma_{1}, \ldots, \sigma_{n}\right), \quad \sigma_{i}=\mu_{i}+i \nu_{i}, \quad i=1, \ldots, n ; \quad \mu_{i}>0,
$$

be the eigenvalues of $E$ taken counting multiplicities so that $\mu_{i}, \nu_{i}$ are the eigenvalues of $M$ and $L$, respectively. Since the group $U$ is real, together with with each complex eigenvalue $\sigma_{i}=\mu_{i}+i \nu_{i}$ there exists a conjugate complex eigenvalue $\sigma_{j}=\mu_{i}-i \nu_{i}$. We denote

$$
\mu=\left(\mu_{1}, \ldots, \mu_{n}\right), \quad \nu=\left(\nu_{1}, \ldots, \nu_{n}\right), \quad|\mu|=\sum_{i=1}^{n} \sigma_{i}=\sum_{i=1}^{n} \mu_{i}>0 .
$$

Let $\Gamma$ be a closed infinitely differentiable surface in $\mathbb{R}^{n}$ enveloping the origin so that each trajectory of the group $\left\{U_{k}, k>0\right\}$ intersects this surface just at one point and in a nontangential direction. Such surfaces will be called admissible. It is easy to show that the class of admissible surfaces is non-empty, in particular, as such surface one can take an ellipsoid sufficiently squeezed along certain axes. In $\mathbb{R}^{n}$ we introduce generalized spherical coordinates by the formula

$$
t=\varrho(r, e)=U_{r} e, \quad e \in \Gamma, r>0 .
$$

Let $\varphi(t) \in S\left(\mathbb{R}^{n}\right)$. Then by transformation (2.5) maps it to the function $\psi(r, e)=\varphi\left(U_{r} e\right)$ defined on $\Gamma \times \mathbb{R}_{+}$. These mappings are denoted by $\zeta$ so that

$$
\zeta: \quad \varphi \mapsto \psi(r, e)=\varphi\left(U_{r} e\right), \quad r \in \mathbb{R}_{+}, \quad e=\left(e_{1}, \ldots, e_{n}\right) \in \Gamma .
$$

There appears a question to which space the function $\psi(r, e)$ belongs? It is easy to see that as $r>0$, the function $\psi(r, e)$ is infinitely differentiable and decays as $r \rightarrow+\infty$ together with all its derivatives faster than each power of $\frac{1}{r}$ and at zero it possesses a special asymptotic expansion. In order to describe the image of the mapping $\zeta$ and to justify the corresponding change of variables, we introduce some definitions.

We let

$$
J_{U}=\left\{\lambda:(\sigma, j)=\lambda, j \in \mathbb{Z}_{+}^{n}\right\},
$$

$\mathcal{E}_{\lambda}$ is the space of polynomials $Q(t)$ homogeneous w.r.t. group $\left\{\mathfrak{H}_{k}, k>0\right\}$ of power $\lambda$ so that $\mathcal{E}_{\lambda}=\left\{Q(t): Q\left(\mathfrak{H}_{k} t\right)=k^{\lambda} Q(t)\right\}$. In the space $\mathcal{E}_{\lambda}$ we define operators $\mathrm{A}_{\lambda}$ acting in accordance with the formulae

$$
\mathrm{A}_{\lambda} Q(t)=\operatorname{grad} Q(t) N t=\sum_{k=1}^{n} \sum_{\ell=1}^{n} t_{\ell} \varepsilon_{k \ell} \frac{\partial Q(t)}{\partial t_{k}}, \quad Q(t) \in \mathcal{E}_{\lambda},
$$


where $\varepsilon_{k \ell}$ are the elements of nilpotent matrix associated with the operator $N$. The operators $\mathrm{A}_{\lambda}$ are nilpotent.

We return back to generalized spherical coordinates. Formal asymptotic expansion $\psi(r, e)=$ $\varphi\left(U_{r} e\right)$ at zero reads as

$$
\psi(r, e)=\varphi\left(U_{r} e\right) \sim \sum_{\lambda \in J} r^{\lambda}\left[C_{\lambda, 0}(e)+\ln r C_{\lambda, 1}(e)+\cdots+\ln ^{n(\lambda)} r C_{\lambda, n(\lambda)}(e)\right] .
$$

Here $C_{\lambda, 0}(e)$ is the trace on $\Gamma$ of a polynomial in the space $\mathcal{E}_{\lambda}$ and

$$
C_{\lambda, m}(e)=\left.\frac{1}{m !} \mathrm{A}_{\lambda}^{m} C_{\lambda, 0}(t)\right|_{t=e \in \Gamma}, \quad m=1, \ldots, n(\lambda),
$$

$n(\lambda)$ are some integers. Let us give a rigorous mathematical meaning to these observations.

Let $\Gamma$ be an admissible surface in $\mathbb{R}^{n}$. The space $S(\Gamma)$ is that of infinitely differentiable on this surface functions with standard topology of uniform convergence together with all the derivatives. The corresponding system of semi-norms is denoted by $Q_{N}\{\cdot\}$.

We introduce the space $W_{\bar{J}_{U}}$ as that of the functions $\psi(r, e)$ being infinitely differentiable as $e \in \Gamma$ and $r \in \mathbb{R}_{+}$, for which as $N=0,1, \ldots$ there exists functions $C_{\lambda, m}(e) \in S(\Gamma), \lambda \in J, 0 \leqslant$ $m \leqslant n(\lambda)$, so that

$$
\begin{aligned}
\psi(r, e)- & \left.\sum_{\substack{\operatorname{Re} \lambda \leqslant N \\
\lambda \in J}} r^{\lambda} \sum_{m=0}^{n(\lambda)} C_{\lambda, m}(e) \ln ^{m} r \in C^{N}([0,+\infty)) \times S(\Gamma)\right), \\
& \left.\left(\frac{d}{d r}\right)^{\ell}\left[\psi(r, e)-\sum_{\substack{\operatorname{Re} \lambda \leqslant N \\
\lambda \in J}} r^{\lambda} \sum_{m=0}^{n(\lambda)} C_{\lambda, m}(e) \ln ^{m} r\right]\right|_{r=0}=0, \quad 0 \leqslant \ell \leqslant N .
\end{aligned}
$$

We introduce the notation

$$
\begin{aligned}
& \bar{\Omega}_{q}[\psi](r, e)=\sum_{\substack{\operatorname{Re} \lambda \leqslant q \\
\lambda \in J}} r^{\lambda} \omega_{\lambda}[\psi](r, e), \quad \Omega_{q}[\psi](r, e)=\sum_{\substack{\operatorname{Re} \lambda<q \\
\lambda \in J}} r^{\lambda} \omega_{\lambda}[\psi](r, e), \\
& \omega_{\lambda}[\psi](r, e)=\sum_{m=0}^{n(\lambda)} C_{\lambda, m}(e) \ln ^{m} r .
\end{aligned}
$$

The topology on $W_{\bar{J}_{U}}$ is defined by the system of norms

$$
\begin{aligned}
\mathcal{P}_{N}(\psi)=\max _{0 \leqslant \ell \leqslant N} \sup _{r>0} Q_{N}\left\{(1+r)^{N}\left(\frac{d}{d r}\right)^{\ell}\left[\psi(r, e)-\eta(r) \bar{\Omega}_{N}[\psi](r, e)\right]\right\} & + \\
& +\max _{\operatorname{Re} \lambda \leqslant N, \lambda \in J} Q_{N}\left\{C_{\lambda, m}(e)\right\} .
\end{aligned}
$$

In $W_{\bar{J}_{U}}$ we define the subspace

$$
V=\left\{\psi(r, e) \in W_{\bar{J}}: \quad C_{\lambda, 0}(e) \in \mathcal{E}_{\lambda}, \quad C_{\lambda, m}(e)=\frac{1}{m !} \mathrm{A}_{\lambda}^{m} C_{\lambda, 0}(e)\right\} .
$$

Here the space of polynomials homogeneous w.r.t. group $\left\{\mathfrak{H}_{k}, k>0\right\}$ of degree $\lambda$ and the space of its traces on $\Gamma$ are identified and denoted by the same symbol $\mathcal{E}_{\lambda}$. The topology in $V$ is induced by that of $W_{\bar{J}_{U}}$. It is easy to see that $V$ is a closed subspace of the space $W_{\bar{J}_{U}}$. We note that the relations $2.9,22.10$ and 2.8 for the functions in $V$ imply the formula

$$
r \frac{d}{d r} \omega_{\lambda}[\psi](r, e)=\mathrm{A}_{\lambda} \omega_{\lambda}[\psi](r, e)
$$


Theorem 2.1. The mapping $\zeta$ defined by the formula (2.6) is an isomorphism of the spaces $S\left(\mathbb{R}^{n}\right)$ and $V$.

This statement allows one to introduce the functional $f_{s}(r, e), r>0, e \in \Gamma$ for each generalized function $f(t) \in S^{\prime}\left(\mathbb{R}^{n}\right)$ by the formula

$$
\left(f_{s}(r, e), \psi(r, e)\right)=(f(t), \varphi(t)), \text { where } \varphi\left(U_{r} e\right)=\psi(r, e) \in V
$$

so that $f_{s}(r, e)$ belongs to $V^{\prime}$. By Hanh-Banach theorem we can continue $f_{s}$ on whole the $W_{\bar{J}_{U}}$. We denote this continuation by $F(r, e)$ and call it generalized spherical representation of the function $f(t) \in S^{\prime}\left(\mathbb{R}^{n}\right)$ so that

$$
(F(r, e), \psi(r, e))=\left(f_{s}, \psi(r, e)\right), \quad \forall \psi(r, e) \in V .
$$

At that,

$$
\begin{aligned}
\left(f\left(U_{\frac{1}{k}} t\right), \varphi(t)\right) & =\frac{1}{\operatorname{det} U_{\frac{1}{k}}}\left(f(t), \varphi\left(U_{k} t\right)\right)= \\
=\frac{1}{\operatorname{det} U_{\frac{1}{k}}}\left(F(r, e), \varphi\left(U_{r k} e\right)\right) & =\frac{\operatorname{det} U_{k}}{k}\left(F\left(\frac{r}{k}, e\right), \varphi\left(U_{r} e\right)\right) .
\end{aligned}
$$

We observe that the generalized spherical representation $F(r, e)$ of the function $f \in S^{\prime}\left(\mathbb{R}^{n}\right)$ is determined non-uniquely. Formula (2.15) yields

Lemma 2.1. Let $\varrho(k)$ be a self-similar function of order $\alpha$. In order a generalized function $f(t) \in S^{\prime}\left(\mathbb{R}^{n}\right)$ to be asymptotically homogeneous at zero w.r.t. self-similar function $\varrho(k)$ along the group of transformations $U=\left\{U_{k}, k>0\right\}$, its generalized spherical representation $F(r, e)$ is necessary and sufficient to be asymptotically homogeneous at zero in $r$ w.r.t. $\rho_{1}(k)$ on $V$, where

$$
\rho_{1}(k)=\frac{k}{\operatorname{det} U_{k}} \rho(k)=k^{1-|\mu|} \rho(k)
$$

Thus, to describe the class $A O_{\rho}^{-U}\left(S\left(\mathbb{R}^{n}\right)\right)$, it is sufficient the class of asymptotically homogeneous generalized functions on the spaces $V \subset W_{\bar{J}_{U}}$. We preface it by a description of asymptotically homogeneous generalized functions at zero on more general special spaces of generalized functions.

\section{Asymptotically homogeneous Generalized FunCtions AS ZERo ON $W_{\bar{J}}, V_{J, \mathcal{F}}$ AND $S\left(\mathbb{R}^{n}\right)$}

Let $J$ be at most countable (probably empty) set of complex number such that in each halfplane $\{\operatorname{Re} z<a: \quad z \in \mathbb{C}, a \in \mathbb{R}\}$ there is at most a finite number of the points in $J$. To each $\lambda \in J$ we associate a nonnegative integer $n(\lambda) \in \mathbb{Z}_{+}$. The sets of pairs of numbers $(\lambda, n(\lambda))$ are indicated by $\bar{J}$ and called admissible sets. We settle that if $n(\lambda)<0$, then $\lambda \notin J$.

By $S_{\bar{J}}$ we denote the space of the functions $\psi(r) \in C^{\infty}\left(\mathbb{R}_{+}\right)$decaying as $r \rightarrow+\infty$ together with all its derivatives and such that for each $N \in \mathbb{Z}_{+}$and some constants $C_{\lambda, m}$ dependent on $\psi$

$$
\left[\psi(r)-\bar{\Omega}_{N}[\psi](r)\right] \in C^{N}([0,+\infty)),\left.\left(\frac{d}{d r}\right)^{\ell}\left[\psi(r)-\bar{\Omega}_{N}[\psi](r)\right]\right|_{r=0}=0
$$


where $\ell=0, \ldots, N$, and

$$
\begin{aligned}
& \Omega_{N}^{J}[\psi](r)=\sum_{\substack{\operatorname{Re} \lambda<N \\
\lambda \in J}} r^{\lambda} \sum_{m=0}^{n(\lambda)} C_{\lambda, m} \ln ^{m} r, \\
& \bar{\Omega}_{N}^{J}[\psi](r)=\sum_{\substack{\operatorname{Re} \lambda \leqslant N \\
\lambda \in J}} r^{\lambda} \sum_{m=0}^{n(\lambda)} C_{\lambda, m} \ln ^{m} r,
\end{aligned}
$$

The superscript $J$ will be omitted if it is clear which $S_{\bar{J}}$ is meant. The topology in $S_{\bar{J}}$ is defined by the system of norms

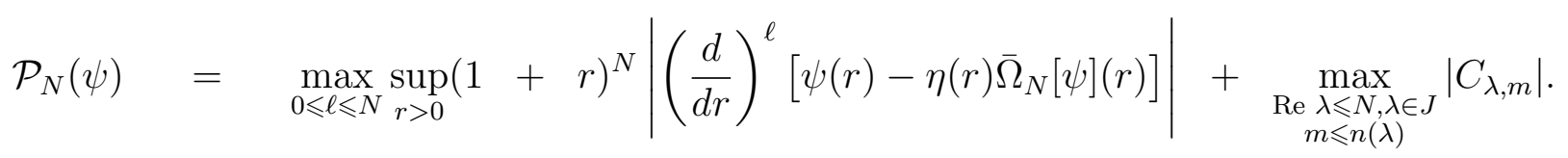

Hereinafter the function $\eta(r)$ is infinitely differentiable on $[0,+\infty)$, compactly supported, and equals 1 in some neighborhood of zero. We observe that at zero $\psi(r) \in S_{\bar{J}}$ has the asymptotic expansion

$$
\psi(r) \sim \sum_{\lambda \in J} r^{\lambda} \sum_{m=0}^{n(\lambda)} C_{\lambda, m} \ln ^{m} r .
$$

The space $S_{\bar{J}}$ is a Frechet space. We also note that $S_{\bar{J}}$ is invariant w.r.t. argument dilation.

As an example of generalized functions in $S_{\bar{J}}^{\prime}$ we adduce the functions

$$
r_{+}^{\beta}, \quad \beta \in \mathbb{C}
$$

generalizing the functions $x_{+}^{\lambda}$ in [2]. In order to do it, we introduce several definitions and notations. Let $\sigma \in J$. By $\overline{J \backslash \sigma}$ we denote the set of pairs $\bar{J}$ with the pair $(\sigma, n(\sigma))$ thrown away, and by $\operatorname{Pr} J$ we indicate the set of real numbers $\{\operatorname{Re} \lambda: \quad \lambda \in J\}$. Let $(\sigma, n(\sigma)) \in \bar{J}$. We define the mapping

$$
D_{\sigma} \equiv r^{\sigma}\left(r \frac{d}{d r}\right)^{n(\sigma)+1} r^{-\sigma}: \quad \varphi(r) \mapsto \psi(r)=D_{\sigma} \varphi(r)
$$

The mapping $D_{\sigma}$ is an isomorphism of the spaces $S_{\bar{J}}$ and $S_{\overline{J \backslash \sigma}}$. We observe that these mapping commutes with dilations.

Let $\gamma \in \mathbb{C}$ and $\bar{J}$ is an admissible set of pais. We let

$$
J_{\gamma}=\{\lambda \in J: \operatorname{Re} \lambda=\operatorname{Re} \gamma\},
$$

It is clear that $J_{\gamma}$ depends only on $\operatorname{Re} \gamma$. We note that $J_{\gamma}$ is a finite set. We let

$$
D_{\bar{J}_{\gamma}}=\left\{\begin{array}{c}
1, \quad \text { if } \operatorname{Re} \gamma \notin \operatorname{Pr} J \\
\prod_{\lambda \in J_{\gamma}} D_{\lambda}, \text { if } \operatorname{Re} \gamma \in \operatorname{Pr} J
\end{array}\right.
$$

where the order in which the operators $D_{\lambda}$ are multiplied is somehow fixed. Further results will be independent of this order. It is easy to see that

$$
D_{\bar{J}_{\gamma}} \sum_{\lambda \in J_{\gamma}} r^{\lambda} \omega_{\lambda}[\psi](r)=0, \quad \text { where } \quad \omega_{\lambda}[\psi](r)=\sum_{m=0}^{n(\lambda)} C_{\lambda, m} \ln ^{m} r .
$$

we denote by $S_{\emptyset}$ the space of test functions in $S_{+}$vanishing at origin together with all its derivatives. 
Lemma 3.1. Let $\bar{J}$ be an admissible set and the number $\beta \in \mathbb{C}$ is so that $-\beta-1 \notin J$. Then there exists the unique homogeneous of degree $\beta$ continuation of $r^{\beta}$ from $S_{\emptyset}$ on $S_{\bar{J}}$. This extensions is defined by the formula

$$
\left(r_{+}^{\beta}, \varphi(r)\right)=\left\{\begin{array}{c}
\int_{0}^{\infty} r^{\beta}\left(\varphi(r)-\bar{\Omega}_{-\operatorname{Re} \beta-1}[\varphi](r)\right) d r \\
\quad \text { if }-\operatorname{Re} \beta-1 \notin \operatorname{Pr} J \\
\prod_{\lambda \in J_{-\beta-1}}\left(\frac{-1}{\beta+1+\lambda}\right)^{n(\lambda)+1} \int_{0}^{\infty} r^{\beta} D_{\bar{J}_{-\beta-1}}\left(\varphi(r)-\bar{\Omega}_{-\operatorname{Re} \beta-1}[\varphi](r)\right) d r, \\
\text { if }-\operatorname{Re} \beta-1 \in \operatorname{Pr} J,
\end{array}\right.
$$

where $\varphi(r) \in S_{\bar{J}}$, and $\Omega_{-\operatorname{Re} \beta-1}[\varphi](r)$ is defined in (3.1).

We note that $r_{+}^{\beta}$ is meromorhpic w.r.t. $\beta \in \mathbb{C}$ generalized function and at the points $-\lambda-$ $1, \lambda \in J$, it has the poles of order $n(\lambda)+1$. So, in a vicinity of the point $\beta_{0}+1 \in-J$ the function $\left(r_{+}^{\beta}, \varphi(r)\right)$ is expanded into the Laurent series

$$
\begin{aligned}
& \left(r_{+}^{\beta}, \varphi(r)\right) \sim \frac{d_{\beta_{0}}}{\left(\beta-\beta_{0}\right)^{n\left(\beta_{0}\right)+1}}+\cdots \\
& \quad \text { where } d_{\beta_{0}}=(-1)^{n\left(-\beta_{0}-1\right)}\left(n\left(-\beta_{0}-1\right)\right) ! C_{-\beta_{0}-1, n(-\beta-1)} .
\end{aligned}
$$

In $S_{\bar{J}}^{\prime}$ we introduce generalized functions

$$
\Delta_{\lambda, m}(r), \quad m=0, \ldots, n(\lambda),
$$

analogues of delta-functions and its derivatives. Suppose $(\lambda, n(\lambda)) \in \bar{J}$ and $\psi(r) \in S_{\bar{J}}$ and let

$$
\left(\Delta_{\lambda, m}(r), \psi(r)\right)=C_{\lambda, m}, \quad m=0, \ldots, n(\lambda),
$$

where $C_{\lambda, m}$ are the corresponding coefficients of expansion (3.2).

Lemma 3.2. Let $\rho(k)$ be a self-similar function of order $\beta$ and $F(r) \in S_{\bar{J}}^{\prime}$ and its support is separated from zero, i.e., there exists a number $a>0$ such that $\operatorname{supp} F(r) \subset\{r \geqslant a\}$. Then $F(r)$ has a trivial quasiasymptotics at zero in $\rho(k)$.

Employing the ideas of works [1] and [5], one can easily prove the following theorems.

Theorem 3.1. Let $\bar{J}$ be an admissible set, $\rho(k)$ be a self-similar function of order $\beta$, at that $\operatorname{Re} \beta-1 \notin \operatorname{Pr} J$, and a number $\ell$ is so that

$$
\operatorname{Re} \beta-1-\operatorname{Re} \ell \notin \mathbb{Z}_{+} .
$$

Then $F(r) \in A O_{\rho}^{-1}\left(S_{\bar{J}}\right)$ if and only if

$$
F(r)=F_{0}(r)+F_{1}(r), \quad F_{0}, F_{1} \in S_{\bar{J}}^{\prime},
$$

where $\operatorname{supp} F_{0}$ is separated from zero and $F_{1}$ is defined as follows. There exist numbers $A, N \in$ $\mathbb{Z}_{+}$and continuous as $r>0$ function $\gamma(r)$ satisfying

$$
\gamma(r) \sim A r^{N+\ell} \rho\left(\frac{1}{r}\right), \quad r \rightarrow+0,
$$

so that for each test function $\varphi \in S_{\bar{J}}$

$$
\left(F_{1}(r), \varphi(r)\right)=\int_{0}^{1} \gamma(r)\left(\frac{d}{d r}\right)^{N}\left[r^{-\ell}\left(\varphi(r)-\bar{\Omega}_{\operatorname{Re} \beta-1}[\varphi](r)\right)\right] d r .
$$


Theorem 3.2. Let $\bar{J}$ be an admissible set, $\rho(k)$ be a self-similar function of order $\beta$, at that $\operatorname{Re} \beta-1 \in \operatorname{Pr} J$, and a number $\ell \in \mathbb{C}$ satisfies condition (3.10). Then $F(r) \in A O_{\rho}^{-1}\left(S_{\bar{J}}\right)$ if and only if identity (3.11) holds, where supp $F_{0}(r)$ is separated from zero and for each $\psi(r) \in S_{\bar{J}}$ the generalized function $F_{1}(r)$ is determined by the formula

$$
\left(F_{1}(r), \psi(r)\right)=\int_{0}^{1} \gamma(r)\left(\frac{d}{d r}\right)^{N} r^{-\ell}\left(D_{\bar{J}_{\beta-1}}\right)\left(\psi(r)-\bar{\Omega}_{\operatorname{Re} \beta-1}[\psi](r)\right) d r
$$

with some $N \in \mathbb{Z}_{+}$and a continuous function $\gamma(r)$ obeying asymptotic relation (3.12). Here Here $D_{\bar{J}_{\beta-1}}$ are determined by formula 3.5 .

Let $\Gamma$ be an admissible surface in $\mathbb{R}^{n}$. The space $S(\Gamma)$ is that of infinitely differentiable on this surface functions with standard topology of uniform convergence together with all the derivatives. Suppose $\Gamma$ is covered by a finite number of charts $U_{\alpha}$, in each of which local coordinates $\xi^{\alpha}=\left(\xi_{1}^{\alpha}, \ldots, \xi_{n-1}^{\alpha}\right)$ are defined. Then the corresponding system of semi-norms is introduced as

$$
Q_{N}\{\varphi(e)\}=\max _{\alpha} \max _{|j| \leqslant N} \sup _{\xi^{\alpha} \in U_{\alpha}}\left|\partial^{j} \varphi(\xi)\right|,
$$

where $j$ is a multi-index, $\partial^{j}$ is the corresponding differential operator.

We let $W_{\bar{J}}=S_{\bar{J}} \otimes S(\Gamma)$ (projective tensor product of the spaces $S_{\bar{J}}$ and $S(\Gamma)$ ). The space $W_{\bar{J}}$ can be realized as the space of functions $\psi(r, e)$ being infinitely differentiable as $e \in \Gamma$ and $r \in \mathbb{R}_{+}$. Thus, for each $N \in \mathbb{Z}_{+}$there exist functions $C_{\lambda, m}(e) \in S(\Gamma), \lambda \in J, 0 \leqslant m \leqslant n(\lambda)$, such that

$$
\begin{aligned}
\left.\psi(r, e)-\bar{\Omega}_{N}[\psi](r, e) \in C^{N}([0,+\infty)) \times S(\Gamma)\right) & \\
& \left.\left(\frac{d}{d r}\right)^{\ell}\left[\psi(r, e)-\bar{\Omega}_{N}[\psi](r, e)\right]\right|_{r=0}=0, \quad 0 \leqslant \ell \leqslant N,
\end{aligned}
$$

where $\bar{\Omega}_{N}[\psi](r, e)$ was introduced in 2.11$)$. The topology on $W_{\bar{J}}$ is defined by the system of semi-norms 2.12). For $\psi(r, e) \in W_{\bar{J}}$ we have asymptotic expansion 2.9)

$$
\psi(r, e) \sim \sum_{\lambda \in J} r^{\lambda} \sum_{m=0}^{n(\lambda)} C_{\lambda, m}(e) \ln ^{m} r=\sum_{\lambda \in J} r^{\lambda} \omega_{\lambda}[\psi](r, e), \quad r \rightarrow 0,
$$

which can be differentiated w.r.t. $r$ arbitrary many times. More precisely, for each $M \in \mathbb{Z}_{+}$ there exists $N \in \mathbb{Z}_{+}$such that

$$
Q_{M}\left\{\left(\frac{\partial}{\partial r}\right)^{\ell}\left(\psi(r, e)-\bar{\Omega}_{N}[\psi](r, e)\right)\right\}=O\left(r^{M}\right), \quad r \rightarrow 0, \quad \ell=0, \ldots, M .
$$

In the space $W_{\bar{J}}$ the most part of statements analogous to that in $S_{\bar{J}}$ holds true. In particular, analogues of Theorems 3.1 and 3.2 .

Let $F(r) \in S_{\bar{J}}^{\prime}$ and $\Phi(e) \in S^{\prime}(\bar{\Gamma})$. Then $F(r) \Phi(e) \in W_{\bar{J}}^{\prime}$ is determined by the formula

$$
(F(r) \Phi(e), \psi(r, e))=\left(F(r),(\Phi(e), \psi(r, e))_{e}\right), \quad \psi(r, e) \in W_{\bar{J}}
$$

Hereinafter the superscript $e$ of $(\Phi(e), \psi(r, e))_{e}$ means the value of the generalized function $\Phi(e) \in S^{\prime}(\Gamma)$ on the function $\psi(r, e)$ treated as a test one in $S(\Gamma)$ for a fixed $r$. 
In particular, if $-\beta-1 \notin J$,

$$
\begin{aligned}
\left(r_{+}^{\beta} \Phi(e), \psi(r, e)\right)= & \left\{\begin{array}{r}
\int_{0}^{\infty} r^{\beta}\left(\Phi(e), \psi(r, e)-\bar{\Omega}_{-\operatorname{Re} \beta-1}[\psi](r, e)\right)_{e} d r, \text { as }-\operatorname{Re} \beta-1 \notin \operatorname{Pr} J \\
C \int_{0}^{\infty} r^{\beta} D_{\bar{J}_{-\beta-1}}\left(\Phi(e), \psi(r, e)-\bar{\Omega}_{-\operatorname{Re} \beta-1}[\psi](r, e)\right)_{e} d r \\
\text { as }-\operatorname{Re} \beta-1 \in \operatorname{Pr} J,
\end{array}\right.
\end{aligned}
$$

where $C=\prod_{\lambda \in J_{-\beta-1}}\left(\frac{-1}{\beta+1+\lambda}\right)^{n(\lambda)+1}$. The function $r_{+}^{\beta} \Phi(e)$ is homogeneous in $r$ of order $\beta$.

Let $\Phi(e) \in S^{\prime}(\Gamma)$, then

$$
\left(\Delta_{\lambda, m}(r) \Phi(e), \psi(r, e)\right)=\left(\Phi(e), C_{\lambda, m}(e)\right)_{e} .
$$

In the space $W_{\bar{J}}$ the generalized functions asymptotically homogeneous at zero in a noncritical case are described by the following theorem.

Theorem 3.3. Let $\bar{J}$ be an admissible set, $\rho(k)$ be a self-similar function of order $\beta$, at that $\operatorname{Re} \beta-1 \notin \operatorname{Pr} J$, and a number $\ell \in \mathbb{C}$ is so that

$$
\operatorname{Re} \beta-\operatorname{Re} \ell-1 \notin \mathbb{Z}_{+} .
$$

Then $F(r, e) \in A O_{\rho}^{-1}\left(W_{\bar{J}}\right)$ if and only if

$$
F(r, e)=F_{0}(r, e)+F_{1}(r, e), \quad F_{0}(r, e), F_{1}(r, e) \in W_{\bar{J}}^{\prime},
$$

where $\operatorname{supp} F_{0}(r)$ is separated from zero, and $F_{1}(r, e)$ is represented as follows. There exists a number $N \in \mathbb{Z}_{+}$and a continuous in $r$ function $\gamma(r, e)$ with values in $S^{\prime}(\Gamma)$ satisfying asymptotic relation

$$
\gamma(r, e) \sim r^{N+\ell} \rho\left(\frac{1}{r}\right) B(e), \quad r \rightarrow+0,
$$

with some generalized function $B(e) \in S^{\prime}(\Gamma)$ such that for each $\psi(r, e) \in W_{\bar{J}}$

$$
\left(F_{1}(r, e), \psi(r, e)\right)=\int_{0}^{1}\left(\gamma(r, e),\left(\frac{d}{d r}\right)^{N}\left(r^{-\ell}\left(\psi(r, e)-\bar{\Omega}_{\operatorname{Re} \beta-1}[\psi](r, e)\right)\right)\right)_{e} d r .
$$

In the space $W_{\bar{J}}$ the generalized functions asymptotically homogeneous at zero in the critical case are described by the following theorem.

Theorem 3.4. Let $\bar{J}$ be an admissible set, $\rho(k)$ be a self-similar function of order $\beta$, at that $\operatorname{Re} \beta-1 \in \operatorname{Pr} J$, and a number $\ell \in \mathbb{C}$ is such that condition (3.19) is satisfied. Then $F(r, e) \in A O_{\rho}^{-1}\left(W_{\bar{J}}\right)$ if and only if

$$
F(r, e)=F_{0}(r, e)+F_{1}(r, e), \quad F_{0}(r, e), F_{1}(r, e) \in W_{\bar{J}}^{\prime},
$$

where $\operatorname{supp} F_{0}(r)$ is separated from zero, and $F_{1}(r, e)$ is represented as follows. There exists a number $N \in \mathbb{Z}_{+}$and a continuous in $r$ function $\gamma(r, e)$ with values in $S^{\prime}(\Gamma)$ satisfying asymptotic relation (3.20) with some generalized function $B(e) \in S^{\prime}(\Gamma)$ such that for each $\psi(r, e) \in W_{\bar{J}}$

$$
\left(F_{1}(r, e), \psi(r, e)\right)=\int_{0}^{1}\left(\gamma(r, e),\left(\frac{d}{d r}\right)^{N} r^{-\ell}\left(D_{\bar{J}_{\beta-1}}\right)\left(\psi(r, e)-\bar{\Omega}_{\operatorname{Re} \beta-1}[\psi](r, e)\right)\right)_{e} d r .
$$

Definition 2. Suppose that to each $\lambda \in J$ a finite-dimensional subspace $\mathcal{E}_{\lambda} \in S(\Gamma)$ and a nilpotent operator $A_{\lambda}$ in $\mathcal{E}_{\lambda}$ are associated so that $A_{\lambda}^{n(\lambda)+1} \varphi \equiv 0$ as $\varphi \in \mathcal{E}_{\lambda}$. Moreover, if $\lambda_{1}, \lambda_{2} \in J$, at that $\operatorname{Re} \lambda_{1}=\operatorname{Re} \lambda_{2}$, but $\operatorname{Im} \lambda_{1} \neq \operatorname{Im} \lambda_{2}$, then

$$
\mathcal{E}_{\lambda_{1}} \cap \mathcal{E}_{\lambda_{2}}=\{0\}
$$


We define a subspace in $W_{\bar{J}}$, letting

$$
V_{J, \mathcal{F}}=\left\{\psi(r, e) \in W_{\bar{J}}: \quad C_{\lambda, 0}(e) \in \mathcal{E}_{\lambda}, \quad C_{\lambda, m}(e)=\frac{1}{m !} A_{\lambda}^{m} C_{\lambda, 0}(e)\right\},
$$

where $\mathcal{F}=\left\{\mathcal{E}_{\lambda}, A_{\lambda}: \lambda \in J\right\}$, and $m=0, \ldots, n(\lambda)$. The topology in $V_{J, \mathcal{F}}$ is induced by that of $W_{\bar{J}}$. It is easy to see that $V_{\bar{J}, \mathcal{F}}$ is a closed subspace of the space $W_{\bar{J}}$.

The next theorem together with Theorem 3.3 provides the description of generalized function in the class $A O_{\rho}^{-1}\left(V_{J, \mathcal{F}}\right)$ for the noncritical case.

Theorem 3.5. Let $\bar{J}$ be an admissible set, $\rho(k)$ be a self-similar function of order $\beta$, at that, $\operatorname{Re} \beta-1 \notin \operatorname{Pr} J$, and $F(r, e) \in W_{\bar{J}}$. If $F(r, e) \in A O_{\rho}^{-1}\left(V_{J, F}\right)$, then $F(r, e)$ is continued to $\widehat{F}(r, e) \in A O_{\rho}^{-1}\left(W_{\bar{J}}\right)$.

To describe asymptotically homogeneous functions at zero in the critical case, we make auxiliary constructions clarifying the structure of the spaces $V_{J, \mathcal{F}}$.

Since $A_{\lambda}$ is nilpotent, in $\mathcal{E}_{\lambda}$ there exists a basis

$$
\left\{\chi_{\ell, m}^{\lambda}(e) \in \mathcal{E}_{\lambda}, \quad \ell=1, \ldots, q_{\lambda} ; \quad m=0,1, \ldots, m_{\ell}^{\lambda}\right\}
$$

such that for each $\ell=1, \ldots, q_{\lambda}$,

$$
A_{\lambda} \chi_{\ell, 0}^{\lambda}(e)=0, \quad A_{\lambda} \chi_{\ell, m}^{\lambda}(e)=\chi_{\ell, m-1}^{\lambda}(e), \quad 1 \leqslant m \leqslant m_{\ell}^{\lambda} .
$$

Let $\psi(r, e) \in V_{\bar{J} \mathcal{F}}$. We fix $\lambda \in J$ and in asymptotic relation (3.16) we separate a term corresponding to this $\lambda$,

$$
\psi(r, e) \sim \cdots+r^{\lambda} \omega_{\lambda}[\psi](r, e)+\ldots, \text { where } \omega_{\lambda}[\psi](r, e)=\sum_{m=0}^{n(\lambda)} \ln ^{m} r \frac{1}{m !} A_{\lambda}^{m} C_{\lambda, 0}(e) .
$$

Expanding $C_{\lambda, 0}(e)$ and $\omega_{\lambda}[\psi](r, e)$ in terms of basis $(3.24)$, it is easy to get

$$
\begin{aligned}
{\left[\omega_{\lambda}[\psi](r, e)\right]_{\ell, m}=\left(r \frac{d}{d r}\right)\left[\omega_{\lambda}[\psi](r, e)\right]_{\ell, m-1}=\cdots=} & =\left(r \frac{d}{d r}\right)^{m}\left[\omega_{\lambda}[\psi](r, e)\right]_{\ell, 0},
\end{aligned}
$$

Let $\gamma \in \mathbb{C}$. We recall that $J_{\gamma}=\{\lambda \in J: \operatorname{Re} \lambda=\operatorname{Re} \gamma\}$. Denote

$$
E_{\gamma}=\bigoplus_{\lambda \in J_{\gamma}} \mathcal{E}_{\lambda}=\operatorname{Lin}\left\{\chi_{\ell, m}^{\lambda}(e): \lambda \in J_{\gamma}, \ell=1, \ldots, q_{\lambda}, 0 \leqslant m \leqslant m_{\ell}^{\lambda}\right\}
$$

Let

$$
\left\{\chi_{\ell, m}^{\lambda *}(e) \in S^{\prime}(\Gamma): \lambda \in J_{\gamma}, \ell=1, \ldots, q_{\lambda}, 0 \leqslant m \leqslant m_{\ell}^{\lambda}\right\},
$$

be a biorthogonal family of generalized functions in $S^{\prime}(\Gamma)$, i.e., the family with the properties

$$
\begin{gathered}
\left(\chi_{\ell, m}^{\lambda *}(e), \chi_{\ell^{\prime}, m^{\prime}}^{\lambda^{\prime}}(e)\right)=\delta_{\ell, \ell^{\prime} ; m, m^{\prime}}^{\lambda, \lambda^{\prime}}, \\
\lambda, \lambda^{\prime} \in J_{\gamma}, \ell^{\prime}=1, \ldots, q_{\lambda^{\prime}}, 0 \leqslant m^{\prime} \leqslant m_{\ell^{\prime}}^{\lambda^{\prime}}, \quad \ell=1, \ldots, q_{\lambda}, 0 \leqslant m \leqslant m_{\ell}^{\lambda},
\end{gathered}
$$

where $\delta_{\ell, \ell^{\prime} ; m, m^{\prime}}^{\lambda, \lambda^{\prime}}$ is the Kronecker delta. The choice of this family is not unique. In what follows we shall use it.

Now we are able to give the description of asymptotically homogeneous functions on $V_{J, F}$ in the critical case.

Theorem 3.6. Let $\bar{J}$ be an admissible set, $\rho(k)$ be a self-similar function of order $\beta$, at that $\operatorname{Re} \beta-1 \in \operatorname{Pr} J$, and let in $W_{\bar{J}}$ there is a subspace $V_{J, \mathcal{F}}$. At that, in $\mathcal{E}_{\lambda}$ we choose a basis $\left\{\chi_{\ell, m}^{\lambda}(e)\right\}$ like in (3.24)-(3.25), and in $S^{\prime}(\Gamma)$ a biorthogonal system $\left\{\chi_{\ell, m}^{\lambda *}(e)\right\}, c f$. (3.28), (3.29). Suppose also that there is a number $\kappa \in \mathbb{C}$ satisfying relation

$$
\operatorname{Re} \beta-1-\operatorname{Re} \kappa \notin \mathbb{Z}_{+} .
$$


Then $F(r, e) \in A O_{\rho}^{-1}\left(V_{J, \mathcal{F}}\right)$ if and only if on $V_{J, \mathcal{F}}$

$$
F(r, e)=F_{0}(r, e)+F_{1}(r, e)+F_{2}(r, e),
$$

where $F_{0}, F_{1}$ and $F_{2}$ obey the following conditions.

$F_{0}(r, e) \in W_{\bar{J}}^{\prime}$, has a support separated from zero.

The generalized function $F_{1}(r, e) \in W_{\bar{J}}^{\prime}$ is defined as follows,

$$
\left(F_{1}(r, e), \psi(r, e)\right)=
$$

$$
=\int_{0}^{1}\left(\gamma_{1}(r, e),\left(\frac{d}{d r}\right)^{N} r^{-\kappa}\left(\psi(r, e)-\Omega_{\operatorname{Re} \beta-1}[\psi](r, e)\right)\right)_{e} d r,
$$

for each $\psi(r, e) \in V_{J, \mathcal{F}}$, with some $N \in \mathbb{Z}_{+}$and continuous in $r>0$ function $\gamma_{1}(r, e)$ with values in $S^{\prime}(\Gamma)$ so that

$$
\begin{gathered}
\left(\gamma_{1}(r, e), \varphi(e)\right) \equiv 0 \text { in } S_{\bar{J}}^{\prime}, \quad \forall \varphi(e) \in E_{\beta-1}=\bigoplus_{\lambda \in J_{\beta-1}} \mathcal{E}_{\lambda}, \\
\gamma_{1}(r, e) \sim r^{N+\kappa} \rho\left(\frac{1}{r}\right) B_{1}(e), r \rightarrow+0, \text { on } S(\Gamma),
\end{gathered}
$$

with some $B_{1}(e) \in S^{\prime}(\Gamma)$.

The generalized function $F_{2}(r, e) \in W_{\bar{J}}^{\prime}$ is represented as follows,

$$
\begin{aligned}
&\left(F_{2}(r, e), \psi(r, e)\right)=\sum_{\lambda \in J_{\beta-1}} \sum_{\ell=1}^{q_{\lambda}} \sum_{m=1}^{m_{\ell}^{\lambda}+1} \int_{0}^{1} \gamma_{\ell, m}^{\lambda}(r)\left(\frac{d}{d r}\right)^{Q} \\
&\left(r^{-\kappa}\left(\chi_{\ell, m}^{\lambda *}(e)-r^{\lambda}\left(r \frac{d}{d r}\right) r^{-\lambda} \chi_{\ell, m-1}^{\lambda *}(e), \psi(r, e)-\bar{\Omega}_{\operatorname{Re} \beta-1}[\psi](r, e)\right)_{e}\right) d r
\end{aligned}
$$

for each $\psi(r, e) \in V_{J, \mathcal{F}}$, with some $Q \in \mathbb{Z}_{+}$and continuous functions $\gamma_{\ell, m}^{\lambda}(r)$ satisfying asymptotic estimates

$$
\gamma_{\ell, m,}^{\lambda}(r) \sim C_{\lambda, m, \ell} r^{Q+\kappa} \rho\left(\frac{1}{r}\right), \quad r \rightarrow+0
$$

with some constants $C_{\lambda, m, \ell}$. Here we suppose that $\chi_{\ell, m_{\ell}^{\lambda}+1}^{\lambda *}(e)=0$.

Let us describe generalized function in $S^{\prime}\left(\mathbb{R}^{n}\right)$ asymptotically homogeneous at zero along the trajectories defined by the multiplicative one-parametric group $\left\{U_{k}=e^{E \ln k}, k>0\right\}$ of linear transformations $\mathbb{R}^{n}$. The generator of this group $E$ is represented by (2.1). Its eigenvalues determine vector $\sigma$, cf. (2.3), with properties (2.4). In the second section we have introduced the notion of generalized spherical representation $F(r, e) \in W_{\bar{J}}^{\prime}$ for the generalized function $f \in S^{\prime}\left(\mathbb{R}^{n}\right)$ so that

$$
(f(t), \varphi(t))=(F(r, e), \psi(r, e)), \quad \psi(r, e)=\varphi\left(U_{r} e\right), \quad r>0, e \in \Gamma
$$

where $\varphi \in S\left(\mathbb{R}^{n}\right)$, and $\Gamma$ is an admissible surface.

Definition 3. We say that the spaces $S_{\bar{J}}, W_{\bar{J}}$ and $V_{J, \mathcal{F}}$ are generated by the group $\left\{U_{k}, k>\right.$ $0\}$, if the function $\psi(r, e)$ belongs to the space $V_{J, \mathcal{F}}$, where $J$ is determined by the formula (2.7) and the numbers $n(\lambda)$ corresponding to each $\lambda \in J$ are evaluated by asymptotic expansion (2.9). At that, the space $\mathcal{E}_{\lambda}$ associated with each $\lambda$ in $J$ is the space of polynomials $Q(t)$ homogeneous w.r.t. group $\left\{\mathfrak{H}_{k}, k>0\right\}$ of degree $\lambda$ so that $V_{J, \mathcal{F}}=V$, where the latter is defined in (2.13). We also say that the space $W_{\bar{J}}$ is chosen optimally, if $A_{\lambda}^{n(\lambda)}$ is non-zero and $A_{\lambda}^{n(\lambda)+1} \equiv 0$. 
Now, taking into consideration relation (2.16), to describe asymptotically homogeneous generalized functions we can employ Theorem 3.3 in the noncritical case and Theorem 3.6 in the critical one.

In the noncritical case the following theorem holds true.

Theorem 3.7. Suppose we are given a self-similar function $\rho(k)$ of order $\alpha$, at that

$$
\operatorname{Re} \alpha-|\mu| \notin \operatorname{Pr} J
$$

and a number $\ell$ so that

$$
\operatorname{Re}(\alpha-|\mu|-\ell) \notin \mathbb{Z}_{+} .
$$

Then $f(t) \in A O_{\rho}^{-U}\left(S\left(\mathbb{R}^{n}\right)\right)$ if and only if

$$
f(t)=f_{0}(t)+f_{1}(t), \quad f_{0}, f_{1} \in S\left(\mathbb{R}^{n}\right)
$$

where supp $f_{0}(t)$ is separated from zero and the generalized function $f_{1}(t)$ is determined as follows. There exist a number $N \in \mathbb{Z}_{+}$, a continuous in $r$ function $\gamma(r, e)$ with values in $S^{\prime}(\Gamma)$ obeying asymptotic condition

$$
\gamma(r, e) \sim r^{N+|\mu|+\ell-1} \rho\left(\frac{1}{r}\right) B(e), \quad r \rightarrow+0,
$$

with some generalized function $B(e) \in S^{\prime}(\Gamma)$ such that

$$
\begin{aligned}
& \left(f_{1}(t), \varphi(t)\right)= \\
& \quad=\int_{0}^{1}\left(\gamma(r, e),\left(\frac{d}{d r}\right)^{N}\left(r^{-\ell}\left(\varphi\left(U_{r} e\right)-\bar{\Omega}_{\operatorname{Re} \alpha-|\mu|}\left[\varphi\left(U_{r} e\right)\right](r, e)\right)\right)\right)_{e} d r .
\end{aligned}
$$

To formulate an appropriate theorem in the critical case, we shall make use of additional constructions.

Let $\mathcal{E}_{\lambda}$ be the space of the polynomials $Q(t)$ homogeneous w.r.t. the group $\left\{\mathfrak{H}_{k}, k>0\right\}$ of degree $\lambda$. Similarly, $\mathcal{E}_{\lambda}^{*}$ is polynomials $P(t)$ homogeneous w.r.t. the group $\left\{\mathfrak{H}_{k}^{T}, k>0\right\}$ transposed to $\mathfrak{H}_{k}$ so that

$$
\mathcal{E}_{\lambda}=\left\{Q(t): Q\left(\mathfrak{H}_{k} t\right)=k^{\lambda} Q(t)\right\}, \quad \mathcal{E}_{\lambda}^{*}=\left\{P(t): P\left(\mathfrak{H}_{k}^{T} t\right)=k^{\lambda} P(t)\right\} .
$$

If a polynomial $Q(t)$ is homogeneous w.r.t. the group $\left\{\mathfrak{H}_{k}, k>0\right\}$ of degree $d=a+i b$, then it is homogeneous w.r.t. the groups $\mathfrak{M}_{k}$ and $\mathfrak{L}_{k}$ of degrees $a$ and $i b$, respectively.

Let $\lambda \in \mathbb{C}$. We denote

$$
E_{\lambda}=\bigoplus_{\substack{\kappa \in J \\ \operatorname{Re} \kappa}} \mathcal{E}_{\kappa}, \quad E_{\lambda}^{*}=\bigoplus_{\substack{\kappa \in J \\ \operatorname{Re} \lambda}} \mathcal{E}_{\kappa}^{*} .
$$

In accordance with said above, all the polynomials in $E_{\lambda}$ are homogeneous w.r.t. the group $\mathfrak{M}_{k}$ of degree Re $\lambda$ (similarly, all the polynomials in $E_{\lambda}^{*}$ are homogeneous w.r.t. the group $\mathfrak{M}_{k}^{T}$ of degree $\operatorname{Re} \lambda$ ). In the spaces $E_{\lambda}$ and $E_{\lambda}^{*}$ we define the operators $\mathrm{A}_{\lambda}$ and $\mathrm{A}_{\lambda}^{+}$acting as

$$
\begin{aligned}
& \mathrm{A}_{\lambda} Q(t)=\operatorname{grad} Q(t) N t=\sum_{k=1}^{n} \sum_{\ell=1}^{n} t_{\ell} \varepsilon_{k \ell} \frac{\partial Q(t)}{\partial t_{k}}, \quad Q(t) \in E_{\lambda}, \\
& \mathrm{A}_{\lambda}^{+} P(t)=t^{T} N^{T}(\operatorname{grad} P(t))^{T}=\sum_{k=1}^{n} \sum_{\ell=1}^{n} t_{\ell} \varepsilon_{\ell k} \frac{\partial P(t)}{\partial t_{k}}, \quad P(t) \in E_{\lambda}^{*},
\end{aligned}
$$

where $\varepsilon_{k \ell}$ are the elements of nilpotent matrix corresponding to the operator $N$. The operators $\mathrm{A}_{\lambda}$ and $\mathrm{A}_{\lambda}^{+}$are nilpotent.

Let

$$
P(t) \in E_{\lambda}^{*}, \quad Q(t) \in E_{\lambda}, \quad \lambda \in J
$$


Employing the chain rule, we obtain

$$
\begin{aligned}
p\left(t^{\prime}\right)=P\left(\frac{\partial}{\partial t^{\prime}}\right) Q\left(t^{\prime}\right)=\left.P\left(\left(\mathfrak{M}_{k}^{T}\right)^{-1} \frac{\partial}{\partial t}\right) Q\left(\mathfrak{M}_{k} t\right)\right|_{t=\mathfrak{M}_{k}^{-1} t^{\prime}}= & \\
& =\left.k^{-\lambda} P\left(\frac{\partial}{\partial t}\right) k^{\lambda} Q(t)\right|_{t=\mathfrak{M}_{k}^{-1} t^{\prime}}=\left.p(t)\right|_{\mathfrak{M}_{k}^{-1} t^{\prime}}=p\left(\mathfrak{M}_{k}^{-1} t^{\prime}\right) .
\end{aligned}
$$

Since $p(t)$ is a polynomial, the matrix of the operator $\mathfrak{M}_{k}$ is non-degenerate and all its eigenvalues are positive that is possible only as $p(t)=$ const. Thus, on $E_{\lambda}^{*} \times E_{\lambda}$ one can introduce a bilinear form

$$
<P(t), Q(t)>=P\left(\frac{\partial}{\partial t}\right) Q(t), \quad P(t) \in E_{\lambda}^{*}, Q(t) \in E_{\lambda}, \quad \lambda \in J .
$$

Let us point out some properties of this bilinear form.

1. If $P \in \mathcal{E}_{\lambda_{1}}^{*}, Q \in \mathcal{E}_{\lambda_{2}}$, and $\lambda_{1} \neq \lambda_{2}$, then $<P(t), Q(t)>=0$.

2. The operators $A_{\lambda}$ and $A_{\lambda}^{+}$are mutually adjoint w.r.t. bilinear form 3.46) so that

$$
<P(t),\left[\mathrm{A}_{\lambda} Q\right](t)>=<\left[\mathrm{A}_{\lambda}^{+} P\right](t), Q(t)>, \quad P(t) \in E_{\lambda}^{*}, \quad Q(t) \in E_{\lambda}, \quad \lambda \in J .
$$

3. The spaces $\mathcal{E}_{\lambda}$ and $\mathcal{E}_{\lambda}^{*}$ are invariant w.r.t. the operators $\mathrm{A}_{\lambda}$ and $\mathrm{A}_{\lambda}^{+}$.

The operators $A_{\lambda}$ and $A_{\lambda}^{+}$can be continued on whole $S(\Gamma)$. In order to do it, we introduce the following definitions.

Definition 4. Suppose we are given a one-parametric group $\mathcal{B}=\left\{\mathcal{B}_{k}, k>0\right\}$, an admissible surface $\Gamma$, and a number $\lambda \in \mathbb{C}$. For each $\varphi(e) \in S(\Gamma)$ we define the continuation operator $\operatorname{cont}_{\mathcal{B}, \lambda}[\varphi](t)$ as a homogeneous w.r.t. the group $\mathcal{B}$ of degree $\lambda$ continuation of the function $\varphi(e)$ from $\Gamma$ on $\mathbb{R}^{n} \backslash\{0\}$.

Definition 5. Suppose we are given a one-parametric group $\mathcal{B}=\left\{\mathcal{B}_{k}, k>0\right\}$, an admissible surface $\Gamma$, a number $\lambda \in \mathbb{C}$, and a generalized function $f(t) \in S^{\prime}\left(\mathbb{R}^{n}\right)$ whose supp $f$ is bounded and separated from zero. We define its restriction on $S(\Gamma)$ by the formula

$$
\left(\text { rest }_{\mathcal{B}, \lambda}[f](e), \varphi(e)\right)=\left(f(t), \operatorname{cont}_{\mathcal{B}, \lambda}[\varphi](t)\right), \quad \varphi(e) \in S(\Gamma) .
$$

We note that if $\varphi(e)$ is the trace of a polynomial homogeneous w.r.t. the group $\mathcal{B}$ of degree $\lambda$, then

$$
\left(\text { rest }_{\mathcal{B}, \lambda}[f](e), \varphi(e)\right)=(f(t), \varphi(t)) .
$$

Let $\left\{\chi_{\ell, m}^{\lambda}(e)\right\}$ be the canonical basis of the operator $A_{\lambda}$ in $\mathcal{E}_{\lambda}$. Then

$$
\left\{\chi_{\ell, m}^{\lambda}(e), \quad \lambda \in J_{\lambda}, 1 \leqslant \ell \leqslant q_{\lambda}, 0 \leqslant m \leqslant m_{\ell}^{\lambda}\right\}
$$

is the canonical basis of the operator $A_{\lambda}$ in $E_{\lambda}$. Let us construct a special biorthogonal family $\left\{\chi_{\ell, m}^{\lambda *}\right\} \in S^{\prime}(\Gamma)$. Let $\left\{\widehat{\chi}_{\ell, m}^{\lambda}(e)\right\}$ be the family of polynomials biorthogonal to $\left\{\chi_{\ell, m}^{\lambda}\right\}$ in $E_{\lambda}^{*}$ w.r.t. bilinear form (3.46) and $g(t) \in S^{\prime}\left(\mathbb{R}^{n}\right)$ be a generalized function with compact support separated from zero so that $(g(t), 1)=1$, for instance, $g(t)=\delta\left(t-t_{0}\right)$, where $t_{0} \in \Gamma$. Then as the family $\left\{\chi_{\ell, m}^{\lambda *}\right\}$ one can take

$$
\chi_{\ell, m}^{\lambda *}(e)=\operatorname{rest}_{\mathfrak{M}_{k}, \operatorname{Re} \lambda}\left[\hat{\chi}_{\ell, m}^{\lambda}\left(\frac{\partial}{\partial t}\right) g(t)\right](e) .
$$

We observe that under such choice

$$
\widehat{\chi}_{\ell, m}^{\lambda}(e)=A_{\lambda}^{+} \widehat{\chi}_{\ell, m+1}^{\lambda}, \quad m=0, \ldots, m_{\ell}^{\lambda}+1,
$$

where, as above, we suppose $\widehat{\chi}_{\ell, m_{\ell}^{\lambda}+1}^{\lambda}(e) \equiv 0$. Now Theorem 3.6 becomes 
Theorem 3.8. Let $\rho(k)$ be a self-similar function of order $\alpha$, at that

$$
\operatorname{Re} \alpha-|\mu| \in \operatorname{Pr} J,
$$

$t_{0} \in \Gamma$, and a number $\ell \in \mathbb{C}$ be such that

$$
\operatorname{Re} \alpha-\operatorname{Re} \ell-|\mu| \notin \mathbb{Z}_{+} .
$$

Then $f(t) \in A O_{\rho}^{-U}(V)$ if and only if

$$
f(t)=f_{0}(t)+f_{1}(t)+f_{2}(t), \quad f_{0}, f_{1}, f_{2} \in V^{\prime},
$$

where $f_{0}, f_{1}, f_{2}$ satisfy the following conditions;

supp $f_{0}(t)$ is separated from zero;

$f_{1}(t)$ is represented as follows. There exist a number $N \in \mathbb{Z}_{+}$and continuous in $r$ function $\gamma_{1}(r, e)$ with values in $S^{\prime}(\Gamma)$ satisfying asymptotic relation

$$
\gamma_{1}(r, e) \sim r^{N+\ell+|\mu|-1} \rho\left(\frac{1}{r}\right) B(e), \quad r \rightarrow+0,
$$

with a generalized function $B(e) \in S^{\prime}(\Gamma)$, and the condition

$$
\left(\gamma_{1}(r, e), \varphi(e)\right) \equiv 0, \quad \text { for all } \varphi(e) \in E_{\alpha-|\mu|},
$$

such that for each $\psi(t) \in S\left(\mathbb{R}^{n}\right)$

$$
\left(f_{1}(t), \psi(t)\right)=\int_{0}^{1}\left(\gamma(r, e),\left(\frac{d}{d r}\right)^{N}\left(r^{-\ell}\left(\psi(r, e)-\bar{\Omega}_{\operatorname{Re} \alpha-|\mu|}[\psi](r, e)\right)\right)\right)_{e} d r .
$$

$\left(f_{2}(t), \psi(t)\right)$ is a linear combination in all $\lambda \in J_{\alpha-|\mu|}$ and all polynomials $P_{\lambda}$ ranging some basis of $\mathcal{E}_{\lambda}^{*}$ of the terms

$$
\begin{aligned}
& \int_{0}^{1} \gamma(r)\left(\frac{d}{d r}\right)^{N} r^{-\ell}\left(\text { rest } _ { \mathfrak { M } , \operatorname { R e } \alpha - | \mu | } \left[\left(\left(\mathrm{A}_{\alpha-|\mu|}^{+} P_{\lambda}\right)\left(\frac{\partial}{\partial t}\right)-\right.\right.\right. \\
& \left.\left.\left.\quad-r^{\alpha-|\mu|}\left(r \frac{d}{d r}\right) r^{-\alpha+|\mu|} P_{\lambda}\left(\frac{\partial}{\partial t}\right)\right) \delta\left(t-t_{0}\right)\right](e),\left(\psi\left(U_{r} e\right)-\bar{\Omega}_{\operatorname{Re} \alpha-|\mu|}[\psi](r, e)\right)\right)_{e} d r
\end{aligned}
$$

where a continuous function $\gamma(r)$ (depending on $\lambda$ and polynom $P_{\lambda}(e)$ ) satisfies asymptotic relation

$$
\gamma(r) \sim b r^{N+|\mu|+\ell-1} \rho\left(\frac{1}{r}\right), \quad r \rightarrow+0,
$$

with some constant $b$ (dependent on $\gamma$ ).

In conclusion of this section we note that the complete description of generalized functions homogeneous w.r.t. the group $U$ is given in the work [10].

\section{ON DIVIDING OF GENERALIZED FUNCTION BY POLYNOMIAL WITH PRESERVING QUASIASYMPTOTIC AND SOME APPLICATIONS}

As the application of above given results we consider the following problem.

Suppose we are given a self-similar function $\rho(k)$ of order $\alpha \in \mathbb{C}$, a polynomial $P(t)$ homogeneous w.r.t. the group $\left\{U_{k}, k>0\right\}$ of degree $q \in \mathbb{C}$, i.e., such that

$$
P\left(U_{k} t\right)=k^{q} P(t),
$$

and a generalized function $g(x) \in A O_{\rho}^{U}\left(S\left(\mathbb{R}^{n}\right)\right)$. When the differential equation

$$
P(\partial) u(x)=g(x)
$$

has a solution $u(x) \in A O_{\rho_{1}}^{U}\left(S\left(\mathbb{R}^{n}\right)\right.$, where $\rho_{1}(k)$ is an appropriate self-similar function? 
To solve such kind of problems, we shall make use of certain results on dividing of a generalized function by a polynomial homogeneous w.r.t. the group $U_{k}$ with preserving the quasiasymptotics.

Lemma 4.1. Let $\Gamma$ be an admissible surface w.r.t. the group $U_{k}$ and $P(e)$ be the trace of a homogeneous (w.r.t. this group) polynomial of degree $q$ on $\Gamma$. Then for each norm $Q_{N}\{\cdot\}$ on $S(\Gamma)$ there exist a norm $Q_{M}\{\cdot\}$ and a constant $C$ such that

$$
Q_{N}\{\varphi(e)\} \leqslant C Q_{M}\{P(e) \varphi(e)\}, \quad \varphi(e) \in S(\Gamma) .
$$

This statement follows from Hörmander classical lemma and the following estimate. Let $\widehat{\varphi}(t)$ be a homogeneous (w.r.t. the group $U_{k}$ ) of degree $q$ continuation of the function $\varphi(e)$ from $\Gamma$ into $\mathbb{R}^{n}$ and

$$
\widehat{Q}_{N}\{\psi(t)\}=\max _{|j| \leqslant N} \sup _{t \in \Gamma^{\varepsilon}}\left|\partial^{j} \psi(t)\right|
$$

is the norm for the functions defined in $\mathbb{R}^{n}$. Here $\Gamma^{\varepsilon}$ is an $\varepsilon$-neighborhood of $\Gamma$. Then there exist constant $C$ and $c$ independent of $\varphi(e) \in S(\Gamma)$ such that

$$
c \widehat{Q}_{N}\{\widehat{\varphi}(t)\} \leqslant Q_{N}\{\varphi(e)\} \leqslant C \widehat{Q}_{N}\{\widehat{\varphi}(t)\} .
$$

It implies

Lemma 4.2. Let $\Gamma$ be an admissible surface, $P(e), \quad e \in \Gamma$ be the trace of a polynomial homogeneous w.r.t. the group $\left\{U_{k}, k>0\right\}$, and $\left\{\gamma(k, e) \in S^{\prime}(\Gamma), k>0\right\}$ be a family of generalized function continuous w.r.t. the parameter $k$, at that,

$$
\gamma(k, e) \underset{k \rightarrow+0}{\longrightarrow} \gamma_{0}(e) \quad \text { in } \quad S^{\prime}(\Gamma) .
$$

Then there exists a family of generalized functions

$$
\left\{\sigma(k, e) \in S^{\prime}(\Gamma), k>0\right\},
$$

weakly measurable w.r.t. the parameter $k$ such that

1. $\sigma(k, e) \underset{k \rightarrow+0}{\longrightarrow} \sigma_{0}(e) i n S^{\prime}(\Gamma)$

2. $P(e) \sigma(k, e)=\gamma(k, e)$.

The proof follows exactly the same lines as that of Lemma 5.1 in [1], one just should employ the previous lemma.

Let $S_{\bar{J}}, W_{\bar{J}}$ and $V_{J, \mathcal{F}}$ be generated by the group $\left\{U_{k}, k>0\right\}$, and the space $W_{\bar{J}}$ is chosen optimally, see Definition 3. Let $P(t)$ be a polynomial homogeneous w.r.t. this group of degree $q$, i.e., $P\left(U_{k} t\right)=k^{q} P(t)$. Then it is homogeneous of the same degree w.r.t. the group $\mathfrak{M}_{k}=e^{\ln k M}$ (the group of dilations along the corresponding axes). Therefore, there exists a multi-index $m \in \mathbb{Z}_{+}^{n}$ such that $q=(\sigma, m)$. Denote by $J+q$ the set $\{\lambda+q, \lambda \in J\}$. Let us prove that $\overline{J+q} \subset \bar{J}$, i.e., $J+q \subset J$ and $n(\lambda) \leqslant n(\lambda+q)$.

Indeed, $P(t)=r^{q} P(e)$, and since $S\left(\mathbb{R}^{n}\right)$ is invariant w.r.t. the multiplication by $P(t), V_{J, \mathcal{F}}$ is invariant w.r.t. the multiplication by $r^{q} P(e)$. We take the function $\psi(r, e) \in V_{J, F}$ such that

$$
\omega_{\lambda}[\psi](r, e)=C_{\lambda, 0}(e)+\cdots+C_{\lambda, n(\lambda)}(e) \ln ^{n(\lambda)} r, \quad C_{\lambda, n(\lambda)}(e) \not \equiv 0 .
$$

Then

$$
\omega_{\lambda+q}[P(t) \psi(t)](r, e)=P(e) C_{\lambda, 0}(e)+\cdots+P(e) C_{\lambda, n(\lambda)}(e) \ln ^{n(\lambda)} r
$$

$$
P(e) C_{\lambda, n(\lambda)}(e) \not \equiv 0
$$

And since $r^{q} P(e) \psi(r, e) \in V_{J, \mathcal{F}}$, then $n(\lambda+q) \geqslant n(\lambda)$. 
The proven statement implies that $W_{\bar{J}}$ is invariant w.r.t. the multiplication by $r^{q} P(e)$. In particular, if $\psi(r, e) \in W_{\bar{J}}$, then $\psi_{1}(r, e)=r^{q} \psi(r, e) \in W_{\bar{J}}$, at that,

$$
C_{\lambda, m}^{\psi_{1}}(e)= \begin{cases}C_{\lambda-q}^{\psi}(e), & \text { if } \lambda-q \in J \\ 0, & \text { if } \lambda-q \notin J\end{cases}
$$

Theorem 4.1. Let $\rho(k)$ be a self-similar function of order $\beta \in \mathbb{C}, P(t)$ be a polynomial homogeneous w.r.t. the group $\left\{U_{k}\right\}$ of degree $q$, and $F(r, e) \in A O_{\rho(k)}^{-1}\left(W_{\bar{J}}\right)$. Suppose also

$$
\operatorname{Re}(\beta-1+q) \notin \operatorname{Pr} J \text {. }
$$

Then there exists a generalized function $\Theta(r, e) \in W_{\bar{J}}^{\prime}$ such that

1. $\Theta(r, e) \in A O_{k^{q} \rho(k)}^{-1}\left(W_{\bar{J}}\right)$;

2. $r^{q} P(e) \Theta(r, e)=F(r, e) \quad$ in $W_{\bar{J}}^{\prime}$,

i.e., for each $\psi(r, e) \in W_{\bar{J}}$

$$
\left(\Theta(r, e), P(e) r^{q} \psi(r, e)\right)=(F(r, e), \psi(r, e)) .
$$

Proof. Employing Theorem 3.3, we have $F=F_{0}+F_{1}$, where the support of $F_{0}$ is separated from zero, and $F_{1}$ is defined by the formula

$$
\left(F_{1}(r, e), \psi(r, e)\right)=\int_{0}^{1}\left(\gamma(r, e),\left(\frac{d}{d r}\right)^{N}\left(r^{-\ell}\left(\psi(r, e)-\bar{\Omega}_{\operatorname{Re} \beta-1}[\psi](r, e)\right)\right)\right)_{e} d r
$$

with $\gamma(r, e)$ satisfying the asymptotic relation

$$
\gamma(r, e) \sim r^{N+\ell} \rho\left(\frac{1}{r}\right) B(e), \quad r \rightarrow+0,
$$

for some generalized function $B(e) \in S^{\prime}(\Gamma)$. We note that one can assume that the support of the generalized function obtained as the result of dividing $F_{0}$ by the polynomial $P$ is also separated from zero. This is why it is sufficient to prove theorem for the function $F_{1}$ only. For $\psi(r, e) \in W_{\bar{J}}$ we let

$$
\begin{aligned}
(\Theta(r, e), \psi(r, e)) & =\int_{0}^{1}\left(\sigma(r, e),\left(\frac{d}{d r}\right)^{N} r^{-\ell-q}\left[\psi(r, e)-\bar{\Omega}_{\beta-1+q}[\psi](r, e)\right]\right)_{e} d r \\
& =\int_{0}^{1}\left(\sigma(r, e),\left(\frac{d}{d r}\right)^{N} r^{-\ell_{1}}\left[\psi(r, e)-\bar{\Omega}_{\beta_{1}-1}[\psi](r, e)\right]\right)_{e} d r
\end{aligned}
$$

where $\beta_{1}=\beta+q, \ell_{1}=\ell+q$. Here the family of generalized functions $\sigma(r, e)$ is the result of dividing $\gamma(r, e)$ by $P(e)$ that is the trace of the polynomial on the surface $\Gamma$. It is well-defined by Lemma 4.2 , and moreover

$$
\sigma(r, e) \sim\left[\frac{B(e)}{P(e)}\right] r^{\ell+N} r^{-q} \rho\left(\frac{1}{r}\right), \quad r \rightarrow+0,
$$

where $\left[\frac{B(e)}{P(e)}\right]$ is a some regularization of the ratio of these functions. We observe that the integral in 4.9 is well-defined. By Theorem 3.3 it implies that $\Theta(r, e) \in A O_{k^{q} \rho(k)}^{-1}\left(W_{\bar{J}}\right)$. Let us 
relation (4.6). Employing that $\sigma(r, e) P(e)=\gamma(r, e)$, we get

$$
\begin{aligned}
& \left(\Theta(r, e), P(e) r^{q} \psi(r, e)\right)=\left(\Theta(r, e), P(e) r^{q} \psi(r, e)\right) \\
& \int_{0}^{1}\left(\gamma(r, e),\left(\frac{d}{d r}\right)^{N} r^{-\ell} r^{-\beta+1-q}\left(r^{q} \psi(r, e)-\bar{\Omega}_{\beta-1+q}\left[r^{q} \psi\right](r, e)\right)\right)_{e} d r
\end{aligned}
$$

Now it is sufficient to see that

$$
\left.\bar{\Omega}_{\beta-1+q}\left[r^{q} \psi\right](r, e)=r^{q} \bar{\Omega}_{\beta-1}[\psi](r, e)\right)
$$

and compare with formula (4.7). The proof is complete.

It implies

Theorem 4.2. Let $\rho(k)$ be a self-similar function of order $\alpha, P(t)$ be a homogeneous w.r.t. the group $U_{k}$ polynomial of degree $q$, and $f(t) \in S^{\prime}\left(\mathbb{R}^{n}\right)$ have quasiasymptotics at zero w.r.t. the group $U$ in $\rho(k)$. If

$$
\operatorname{Re}(\alpha-|\mu|+q) \notin \operatorname{Pr} J
$$

there exists a generalized function $u(t) \in S^{\prime}\left(\mathbb{R}^{n}\right)$ having quasiasymptotics at zero w.r.t. the group $U$ in $\rho_{1}(k)=k^{q} \rho(k)$ such that

$$
P(t) u(t)=f(t) .
$$

This theorem is a reformulation of Theorem 4.1, where one should assume $\psi(r, e) \in V$ and employ that if $\psi(r, e) \in V$, then $r^{q} P(e) \psi(r, e) \in V$. Now the answer to the question posed in the beginning of the section is given by

Theorem 4.3. Let $\rho(k)$ be a self-similar function of order $\alpha, P(t)$ be a homogeneous w.r.t. the group $\left\{U_{k}, k>0\right\}$ polynomial of degree $q$ and $g(x) \in A O_{\rho}^{U^{T}}\left(S\left(\mathbb{R}^{n}\right)\right)$.

If

then the equation

$$
\operatorname{Re}(\alpha+q) \notin \operatorname{Pr} J
$$

$$
P(\partial) u(x)=g(x)
$$

has a solution

$$
u(x) \in A O_{\rho_{1}}^{U^{T}}\left(S\left(\mathbb{R}^{n}\right)\right)
$$

where $\rho_{1}(k)=k^{q} \rho(k)$.

The statement of the theorem is in fact the previous theorem formulated in terms of Fourier transform.

Corollary 4.1. If under the hypothesis of the theorem $g(x)$ is homogeneous w.r.t. the group $U^{T}$ of degree $\alpha$, i.e.,

$$
g\left(U_{k}^{T} x\right)=k^{\alpha} g(x)
$$

then the equation (4.16) has a homogeneous w.r.t. the group $U^{T}$ solution of degree $\alpha+q$.

Indeed, according to Theorem 4.3, equation 4.16 has a solution $u(x) \in A O_{k^{\alpha+q}}^{U^{T}}\left(S\left(\mathbb{R}^{n}\right)\right)$. Since the polynomial $P(t)$ is homogeneous w.r.t. the group $\left\{U_{k}, k>0\right\}$ of degree $q$, we have

$$
P(\partial) u\left(U_{k}^{T} x\right)=k^{\alpha+q} g(x) .
$$

It implies

$$
P(\partial) \frac{1}{k^{\alpha+q}} u\left(U_{k}^{T} x\right)=g(x) .
$$

Since by the theorem there exists

$$
\frac{1}{k^{\alpha+q}} u\left(U_{k}^{T} x\right) \underset{k \rightarrow \infty}{\longrightarrow} u_{0}(x)
$$


and $u_{0}(x)$ is homogeneous function, passing to the limit in (4.18), we obtain

$$
P(\partial) u_{0}(x)=g(x) .
$$

Example 1. Suppose in $\mathbb{R}^{4}$ the group

$$
U_{k}=k\left(\begin{array}{cccc}
\cos \tau & -\sin \tau & 0 & 0 \\
\sin \tau & \cos \tau & 0 & 0 \\
0 & 0 & \cos \tau & -\sin \tau \\
0 & 0 & \sin \tau & \cos \tau
\end{array}\right)\left(\begin{array}{cccc}
1 & 0 & \tau & 0 \\
0 & 1 & 0 & \tau \\
0 & 0 & 1 & 0 \\
0 & 0 & 0 & 1
\end{array}\right)
$$

acts, where $\tau=\ln k$. We note that in the variables $z_{1}=t_{1}+i t_{2}, z_{2}=t_{3}+i t_{4}, z_{3}=\bar{z}_{1}, z_{4}=\bar{z}_{2}$, the matrix of this group generator has a standard complex Jordan form

$$
E=\left(\begin{array}{cccc}
1+i & 1 & 0 & 0 \\
0 & 1+i & 0 & 0 \\
0 & 0 & 1-i & 1 \\
0 & 0 & 0 & 1-i
\end{array}\right)
$$

so that $J=\{0,1+i, 1-i, 2, \ldots\}$, in particular, $\operatorname{Pr} J=\mathbb{Z}_{+}$and $|\mu|=4$. It is easy to check that the polynomial $P(t)=t_{2} t_{3}-t_{1} t_{4}$ is homogeneous w.r.t. the group $\left\{U_{k}\right\}$ of degree $q=2$.

Consider an ultrahyperbolic equation

$$
\frac{\partial^{2} u}{\partial x_{3} \partial x_{2}}-\frac{\partial^{2} u}{\partial x_{1} \partial x_{4}}=g(x)
$$

Let $g(x) \in A O_{\rho}^{U^{T}}\left(S\left(\mathbb{R}^{n}\right)\right)$, where $\rho(k)$ is a self-similar function of order $\alpha \in \mathbb{C}$, and

$$
U_{k}^{T}=k\left(\begin{array}{cccc}
\cos \tau & \sin \tau & 0 & 0 \\
-\sin \tau & \cos \tau & 0 & 0 \\
\tau \cos \tau & \tau \sin \tau & \cos \tau & \sin \tau \\
-\tau \sin \tau & \tau \cos \tau & -\sin \tau & \cos \tau
\end{array}\right), \quad \tau=\ln k
$$

If $\operatorname{Re} \alpha+2 \notin \mathbb{Z}_{+}$, in accordance with Theorem 4.3 , there exists a solution $u(x) \in$ $A O_{k^{2} \rho(k)}^{U^{T}}\left(S\left(\mathbb{R}^{n}\right)\right)$. In particular, if $g(x)=\delta(x)$ and $\rho(k)=k^{-4}$, there exists a fundamental solution to equation (4.22) possessing quasiasymptotics in $\rho(k)=k^{-2}$ along the trajectories defined by group 4.23). Moreover, according to Corollary of Theorem 4.3, there exists a fundamental homogeneous w.r.t. group (4.23) of degree -2 solution of equation (4.22). These solutions are the generalized functions

$$
u(x)=\left(x_{2} x_{3}-x_{1} x_{4}+i 0\right)^{-1} \quad \text { or } \quad\left(x_{2} x_{3}-x_{1} x_{4}-i 0\right)^{-1}
$$

determined in [2] (up to multiplicative constants).

Example 2. Consider the equation

$$
\left(\frac{\partial^{2}}{\partial x_{3} \partial x_{2}}-\frac{\partial^{2}}{\partial x_{1} \partial x_{4}}\right)^{2} u(x)=\left(\frac{\partial^{2}}{\partial x_{3}^{2}}+\frac{\partial^{2}}{\partial x_{4}^{2}}\right) \delta(x) .
$$

It is easy to check that the function in the right hand side is homogeneous w.r.t. group (4.23) of degree $\alpha=-6$. Noting that the polynomial $P(t)=\left(t_{2} t_{3}-t_{1} t_{4}\right)^{2}$ is homogeneous w.r.t. the group 4.20 of degree $q=4$, by Corollary of Theorem 4.3 we obtain that there exists a solution to equation (4.25) homogeneous w.r.t. group 4.23) of degree $\alpha+q=-2$.

\section{BIBLIOGRAPHY}

1. Yu.N. Drozhzhinov, B.I. Zavialov. Asymptotically quasi-homogeneous generalized functions at the origin // Ufimskii matem. zhurn. 2009. V. 1, No. 4. P. 33-66. (in Russian).

2. I.M. Gel'fand, G.E. Shilov. Generalized Functions: Properties and operations. Moscow, Fizmatlit, 1959. [Academic Press, N.Y., 1964.] 
3. Yu. N. Drozhzhinov, B.I. Zavialov. Generalized functions asymptotically homogeneous along special transformation groups // Matem. sbornik. 2009. V. 200, No. 6. P. 23-66. [Sb. Math. 2009. V. 200, No. 6. P. 803-844]

4. Yu. N. Drozhzhinov, B.I. Zavialov. Asymptotically homogeneous generalized functions at zero and convolution equations with kernels quasi-homogeneous polynomial symbols // Dokl. RAN. 2009. V. 426, No. 3. P. 300-303. [Dokl. Math. 2009. V. 79, No. 3. P. 356-359.]

5. Yu. N. Drozhzhinov, B.I. Zavialov. Distributions asymptotically homogeneous along the trajectories determined by one-parameter groups // Izv. RAN, ser. matem. 2012. V. 76, No. 3. P. 39-91. [Izv. math. 2012. V. 76, No. 3. P. 466-516.]

6. Eu. Seneta. Regularly varying functions. Lecture Notes in Math. V. 508. Springer-Verlag, Berlin. 1976.

7. V.S. Vladimirov, Yu. N. Drozhzhinov, B.I. Zavialov. Multidimensional Tauberian theorems for generalized functions. Nauka, Moscow. 1986. (in Russian).

8. O. Grudzinski Quazihomogeneous Distribution. North-Holland mathematics studies 165, NorthHolland-Amsterdam, 1991.

9. L. Hörmander On the division of distribution by polynomials // Ark. math. 1958. V. 3, No. 6. P. 555-568.

10. Yu.N. Drozhzhinov, B.I. Zavialov Homogeneous Generalized Functions with Respect to OneParametric Group // p-Adic Numbers, Ultrametric Analysis and Applications. 2012. V. 4, No 1. P. 20-31.

Yurii Nikolaevich Drozhzhinov,

Steklov Mathematical Institute of RAS,

Gubkina, 8,

119991, GSP-1, Moscow, Russia

E-mail: drozzin@mi.ras.ru

Boris Ivanovich Zavialov,

Steklov Mathematical Institute of RAS,

Gubkina, 8,

119991, GSP-1, Moscow, Russia

E-mail: bzavial@mi.ras.ru 